\title{
La dependencia del ejercicio físico en la mujer físicamente activa
}

\author{
Einar Kjelsås, MA* \\ Liv Berit Augestad, $\mathrm{PhD}^{\text {** }}$ \\ K. Gunnar Götestam, MD, PhD* \\ * Departamento de Psiquiatría y Medicina \\ de la Conducta, Facultad de Medicina, \\ Universidad Noruega de Ciencias y \\ Tecnología, Trondheim \\ ** Programa de Ciencias del Deporte, \\ Facultad de Ciencias Sociales y Dirección \\ de la Tecnología, Universidad Noruega de \\ Ciencia y Tecnología, Trondheim
}

NORUEGA

RESUMEN - Antecedentes: El ejercicio y el deporte pueden mejorar la calidad de vida. Sin embargo, algunos ejercicios habituales no siempre asocian la actividad física con la sensación de algo bien hecho o con la promoción de la salud. ¿Cuando el ejercicio físico empieza a tener un aspecto negativo sobre la salud mental y física?

Método: El objeto del estudio fue el analizar las características de la dependencia del ejercicio entre 1221 mujeres noruegas físicamente activas, utilizando el Cuestionario de la Dependencia del Ejercicio. Se analizó la relación entre las horas semanales de ejercicio y la puntuación en el cuestionario.

Resultados: Los resultados mostraron que los dos predictores de la alta actividad física tenían las mas altas puntuaciones en "interferencias con la vida social" y en "síntomas de abstinencia”. Se encontró una fuerte relación entre las horas empleadas para la actividad física y la puntuación conseguida en el cuestionario.

Conclusiones: La tarea de resolver el aspecto disfuncional de la actividad física representa todavía una discusión en desarrollo. Un objetivo razonable para los atletas, entrenadores, terapeutas y profesionales de la salud sería asegurar la continuidad del ejercicio físico sin los aspectos negativos propios de una adicción o dependencia.

\section{Introducción}

La actividad física y el deporte pueden mejorar la calidad de vida (Blair y McCloy
1993, Paffenbarger y Hyde 1989, Petruzello et al. 1991). Durante los años años 80, el nivel de actividad se incrementó, tanto para las personas mayores como para los jóvenes 
en Noruega y en EEUU et al. Danielsen 1989, Benyo 1993). La información obtenida de reuniones médicas muestran que la actividad física incluye factores beneficiarios de la salud (Bahr et al. 1993). Sin embargo, uno no debería mostrarse indiferente al hecho de que la continuidad del ejercicio podría llegar a tener efecto psicológicos y físicos negativos.

Para algunos deportistas habituales, la motivación que lleva al ejercicio, no está tan asociada a la sensación de estar haciendo algo bueno o saludable (Szabo 1995, Pierce 1993, Skaarderud 1994). En nuestras reuniones diarias, a menudo hablamos acerca de " adictos al ejercicio", pero no necesariamente en el aspecto negativo del abuso del ejercicio, que podría llegar a controlar la vida diaria de una persona.

Desde 1970 (Backeland 1970) se han publicado algunos estudios en EEUU, Canadá e Inglaterra, centrados en el exceso del ejercicio (Pierce 1990, 1993, Davis y Fox 1993, Thornton y Scott 1995, Adams y Kirkby 1998, Davis et al. 1999). Los autores han utilizado diferentes expresiones para denominar este fenómeno. Ha habido varios intentos de describir la "dependencia al ejercicio", pero muchas cuestiones han quedado sin resolver (Veale 1991, Yates et al. 1994, Szabo 1995, Davis 2000). Se ha sugerido que la dependencia al ejercicio podría ser análoga a la Anorexia Nerviosa (AN) (Yates et al. 1983). Otros han sugerido que la "dependencia del ejercicio" debería verse como un trastorno mental distinto, o que ambos, la Anorexia Nerviosa y la "dependencia del ejercicio", son variantes de un trastorno compulsivo-obsesivo o de un trastorno spectrum (Hollander 1997). Fenómenos como el abuso del alcohol y de las drogas y recientemente también del juego, se clasifican como adiciones en el DSM-IV americano (APA 1994). Veale (1987) ha sugerido una definición para la "dependencia del ejercicio". Hoy en día, todavía no se ha establecido consenso relacionado con el marco teórico, el criterio de definición y los rasgos clínicos de la "dependencia del ejercicio", sin embargo, en el presente artículo si que hemos descrito la condición en términos de alta puntuación en el cuestionario EDQ.

El principal objetivo de este estudio fue el analizar las puntuaciones del Cuestionario de la Dependencia del Ejercicio entre 1221 mujeres físicamente activas. Hemos tenido en cuenta esta cuestión: ¿Difieren las mujeres con alta actividad física (medido en horas semanales) en cuanto a altas puntuaciones en el Cuestionario de Dependencia del Ejercicio de las mujeres con baja actividad física?

\section{Material y Método}

\section{Sujetos}

Un número total de 1.221 mujeres devolvieron los cuestionarios completados. La muestra consistió en dos grupos: 1)estudiantes universitarios, 2) participantes en competiciones deportivas.

Las estudiantes fueron seleccionadas de entre cuatro universidades noruegas, en Oslo, Bergen, Tromso y Trondheim. Las administraciones de estas universidades dieron su permiso para la realización del estudio. El objetivo era conseguir la respuesta de 1000 estudiantes mujeres, 250 de cada Universidad. Los cuestionarios los completó, voluntariamente, cada estudiante en su propia mesa de trabajo al comienzo del día. Cuando estaban listos, los estudiantes devolvían sus cuestionarios y los introducían en una caja situada fuera de la sala de lecturas, donde la secretaria de la investigación 
se encontraba sentada. Estos estudiantes podrían participar en una lotería. Un total de 803 (80\%) de las mujeres estudiantes, devolvieron el cuestionario.

El grupo de participantes en competiciones deportivas se formó por las mujeres que participaron en el Maratón de Oslo de 1997, junto a las mejores atletas en cuatro deportes individuales y cuatro deportes de equipo. El Maratón de Oslo de 1997 contó con un total de 5.000 participantes, de los cuales 1.000 eran mujeres y 4.000 hombres. Debido a las limitadas fuentes financieras, el cuestionario fue únicamente enviado a 500 de las 1.000 participantes. La muestra fue elegida aleatoriamente. A una de cada cinco personas que completó la carrera, se le envió un cuestionario a su dirección, con sobre y sello para incentivar su respuesta. Esto se realizó en colaboración con la Alianza Bislett, responsable de la organización original. Un criterio para la inclusión era la residencia en Noruega, así como una edad comprendida entre los 18 y 60 años. Ya que los participantes del estudio debían mantenerse en el anonimato, ninguna carta recordatoria se envió. Los cuestionarios eran únicamente enviados a participantes en la mitad del maratón $(21 \mathrm{~km})$ porque solamente 82 mujeres habían competido en una maratón completo (42 km). De las 500 mujeres que habían en origen, solo $236(47,2 \%)$ devolvieron el cuestionario completo. Diez fueron enviados erróneamente a otra dirección y fueron devueltos al remitente. Así que de los 500 cuestionarios enviados a dichas participantes del medio maratón, tuvimos que descontar 10 teniendo como respuestas definitivas las de los 239 sujetos.

Definimos al "top atleta femenino" como una atleta que tuviera altas puntuaciones en deportes durante el año 1997. El cuestionario se envió a 60 atletas que participaron en sky nórdico de travesía, marcha de orienta- ción, carreras de grandes distancias y baile. Cincuenta y tres atletas $(88,3 \%)$ de volvieron el cuestionario completado. Se eligieron cuatro deportes de equipo: balonmano, hockey sobre hielo, fútbol y volleyball. El cuestionario se envió a 232 atletas de deportes en equipo, y de éstos, 129 (55,6\%) completaron el formulario. En resumen, que de un total de 292 cuestionarios enviados a mujeres atletas, 182 fueron devueltos $(62,3 \%)$. La muestra total del estudio consistió en 1.221 mujeres.

\section{Cuestionarios}

Se preparó para el estudio un inventario que incluía cuestiones acerca de la edad, el estado civil, la ocupación principal, las horas semanales de entrenamiento, la altura y el peso. La altura y el peso, fueron convertidos en el Indice de Masa Corporal de Quetelet (BMI, peso en kilogramos dividido por la altura elevada al cuadrado, Van Itallie 1987). Además se utilizó el Cuestionario de la Dependencia del Ejercicio.

Ogden et al. (1997) desarrolló el Cuestionario de la Dependencia del Ejercicio (EDQ) con 29 preguntas diseñadas para valorar los diferentes aspectos de este tipo de dependencia. Cada una se responde de acuerdo a una escala Likert de siete puntos, donde el 1 significa "fuertemente en desacuerdo". El EDQ consta de las siguientes 8 subescalas: Ejercicio por razones de salud (ESR), Ejercicio por razones sociales (ESR), Ejercicio por control de peso (EWC), Percepción de problemas (IP), Interferencia con la vida social (ISL), Gratificación positiva (PR), Comportamiento estereotipado (SB) y Síntomas de abstinencia (WS).

El EDQ es una escala multidimensional que contiene aspectos tales como efectos sociales del ejercicio, factores de motiva- 
ción, y variables emocionales. Se ha validado contra el Test de Actitudes ante la Comida (EAT-26, Garner et al. 1982) y el Perfil del Estado de Humor, POMS, McNair et al. 1971). Cockerill (1996) ha dicho de esta escala que se trata de una buena herramienta para medir la dependencia del ejercicio.

\section{Definición de la dependencia del ejercicio}

La dependencia del ejercicio se percibe en el EDQ en términos de un modelo continuo de comportamiento que mide el nivel de dependencia. Las características en el EDQ tienen mucho en común con las descripciones de otras formas de comportamiento adictivo (Oxford 1984, Marlatt y Gordon 1985). La dependencia del ejercicio se define por Ogden et al. (1997) como una combinación de características biomédicas parecidas a las de las adicciones tales como síntomas de abstinencia y comportamiento estereotipado además de otros aspectos psicosociales como la interferencia con la vida social/familiar y las gratificaciones positivas. Además, la dependencia del ejercicio refleja la motivación de continuar haciendo ejercicio en base al deseo de controlar la línea y el peso, la necesidad de contacto social, y la búsqueda de la salud física. También refleja un reconocimiento de un problema de conducta subjetivamente percibido por el sujeto.

Por el momento, no se ha establecido un consenso acerca de la estructura teórica, del criterio para su definición y de los rasgos clínicos propios de esta dependencia del ejercicio. Como consecuencia de ello, hemos querido describir en el presente estudio, la posible dependencia del ejercicio en relación con las altas puntuaciones en el EDQ, ya que dicha dependencia del ejercicio se explica en términos del EDQ.

\section{Estadística}

Los análisis de fiabilidad interna (Cronbach's alpha) se basaron en factores y en el EDQ total. El ANOVA de una vía (t-test) se utilizó para comparar las puntuaciones del EDQ entre mujeres con una grado de actividad física bajo y mujeres con un grado de actividad física alto. La regresión múltiple paso a paso se utilizó para analizar los predictores de alta actividad física utilizando la variable contínua horas de ejercicio por semana como una variable dependiente. Las probabilidades por debajo de $\mathrm{p}=0,001 * * *$, $p=0,01$ y $p=0,05$ se consideraban significativas y fueron incluidas en el modelo.

\section{Resultados}

\section{Datos demográficos}

Ambos grupos estaban formados por mujeres físicamente activas. Los datos demográficos de todos los participantes $(\mathrm{N}=1221)$ se muestran en la tabla I. Respecto a la muestra total, el $33,6 \% \quad(\mathrm{~N}=410)$ habían dado a luz, el 2,1 ( $\mathrm{N}=25)$ estaban embarazadas o en periodo de lactancia, y el 24\% ( $\mathrm{N}=293)$ tenían responsabilidad sobre niños menores de 18 años.

La edad media del total de la muestra era de 28 años, que era relativamente alta. Esto se debe probablemente a las corredoras de larga distancia de la muestra, cuya media de edad era de 40 años (rango de 19 a 60). La edad media de los estudiantes era de 23 años (rango de 19 a 49) y entre las "atletas top" era de 23 años (rango de 18 a 36). Del total de la muestra, el 10,6\% ( $=131)$ tenían mas de 40 años, y el 20,6\% (N=252) trabajaban. 
Tabla I

Características demográficas de la muestra total $(\mathrm{n}=1221)$ y de las mujeres con alta y baja actividad física

\begin{tabular}{|c|c|c|c|c|c|c|c|c|}
\hline \multirow[t]{2}{*}{ Variable } & \multicolumn{2}{|c|}{$\begin{array}{l}\text { Mujeres } \\
\mathrm{n}=1221\end{array}$} & \multicolumn{2}{|c|}{$\begin{array}{l}\text { Alta act. física } \\
\quad \mathrm{n}=135\end{array}$} & \multicolumn{2}{|c|}{$\begin{array}{l}\text { Baja act. física }{ }^{\mathrm{b}} \\
\mathrm{n}=1057\end{array}$} & \multicolumn{2}{|c|}{$\begin{array}{c}\text { Significación } \\
\text { de los tests }\end{array}$} \\
\hline & Media & (SD) & Media & (SD) & Media & (SD) & $\mathrm{t}$ & $\mathrm{p}$ \\
\hline Edad & 28 & $(8,39)$ & 26 & $(7,50)$ & 27 & $(8,49)$ & 1,79 & NS \\
\hline Altura & 169 & $(5,91)$ & 169 & $(6,32)$ & 168 & $(5,80)$ & 2,46 & $*$ \\
\hline Peso & 63 & $(25,21)$ & 64 & $(16,83)$ & 62 & $(7,37)$ & 2,62 & $* * *$ \\
\hline Índice de masa corporal & 21,7 & $(8,56)$ & 22,2 & $(5,72)$ & 21,7 & $(2,19)$ & 1,53 & NS \\
\hline $\begin{array}{l}\text { Horas de entrenamiento } \\
\text { por semana }\end{array}$ & 5 & $(3,45)$ & 12 & $(3,18)$ & 4 & $(2,15)$ & 39,18 & $* * *$ \\
\hline $\begin{array}{l}\text { Horas de entrenamiento en } \\
\text { estudios de salud por semana }\end{array}$ & 1,4 & $(2,78)$ & 3,8 & $(4,33)$ & 1,2 & $(2,07)$ & 7,08 & $* * *$ \\
\hline Sesiones de trabajo por semana & a $2-3$ & $(1,47)$ & $5-6$ & $(1,82)$ & $2-3$ & $(1,27)$ & 16,34 & $* * *$ \\
\hline Estado civil & $\mathrm{N}$ & $(\%)$ & $\mathrm{N}$ & $(\%)$ & $\mathrm{N}$ & $(\%)$ & $\chi^{2}$ & $\mathrm{p}$ \\
\hline Sola & 760 & $(62,7)$ & 86 & $(64,2)$ & 657 & $(62,3)$ & 0,17 & NS \\
\hline Casada & 175 & $(14,4)$ & 14 & $(10,4)$ & 157 & $(14,9)$ & 1,91 & NS \\
\hline Vive junto a $\ldots$ & 277 & $(22,9)$ & 34 & $(25,4)$ & 240 & $(22,8)$ & 0,45 & NS \\
\hline
\end{tabular}

(*) $\mathrm{p}<, 05$.

$(* *) \mathrm{p}<, 01$.

$(* * *) \mathrm{p}<, 001$

$\mathrm{NS}=$ no significativo.

(a) 10 horas o más por semana.

(b) Menos de 10 horas por semana.

El Indice de Masa Corporal se sitúa entre 15,2 y 31,6 . Entre los estudiantes, la media de BMI era de 21,8 (rango del 15,2 al 31,6), y para grupos participantes en deportes, el BMI se situaba en un 21,6 (rango del 16,1 al 28,2 ) para el corredor, y en un 22,0 (rango del 16,1 al 28,2) para atletas competitivos.

\section{Fiabilidad Interna}

En este estudio, el análisis de fiabilidad interna fue primero realizado sobre factores y con el EDQ total, utilizando el alfa de Cronbach. La puntuación alfa se muestra en la tabla II. La puntuación del EDQ total contaba con un alfa de Cronbach de 0,82. Las puntuaciones alfa de Cronbach sugerieron una fiabilidad interna sólida de los factores y del EDQ total. El "ejercicio por razones sociales" y "la percepción del problema" mostraron la fiabilidad interna mas baja.

\section{Actividad física}

La media de las horas de ejercicio físico de estas mujeres era de 5 horas por semana. Los análisis se llevaron a cabo en dos grupos de "alta actividad física en mujeres" con 5 horas o más y con 10 horas por semana respectivamente. Para la comparación de los grupos se definió como "actividad física baja" a las que hacían menos de 5 horas y menos de 10 horas por semana, respectivamente. Entre las mujeres que ejercían 10 horas o mas por semana, el Indice de Masa Corporal se situó entre un 16,1 y un 27,3, y para aquellos que ejercitaban menos de 10 horas por semana, el rango se situó entre el 15,1 y el 31,6. Esta diferencia no era significativa. 


\section{Puntuaciones EDQ}

El grupo de 5 horas de alta actividad física $(\mathrm{t}=11,88, \mathrm{p}<0,001)$ y el grupo de 10 horas $(\mathrm{t}$ $=5,82, \mathrm{p}<0,001)$ dieron puntuaciones EDQ mas altas que los grupos de baja actividad física. El grupo de 5 horas tenía puntuaciones EDQ mas bajas, comparadas con las mujeres con baja actividad física en ejercicio "por razones de salud" ( $t=5,22, \mathrm{p}<0,001)$ y en "ejercicio para el control del peso" $(t=2,14, p$ $<0,05)$ y mas altas puntuaciones para el resto de los factores con la excepción de "gratificaciones positivas" en donde no se encontraron resultados significativos (tabla II).

El grupo de 19 horas tenía una puntuación similar al grupo de 5 horas, pero el grupo de 10 horas tenía también puntuaciones mas bajas en "gratificaciones positivas" $(\mathrm{t}=-3,45, \mathrm{p}<0,01)$. El grupo de 10 horas tenía todavía medias mas bajas que el grupo de 5 horas en "ejercicio por razones de salud" ( $\mathrm{t}=-8,86, \mathrm{p}<0,001)$ y en "ejercicio para el control del peso" $(\mathrm{t}=-3,95, \mathrm{p}<$ $0,001)$ En "interferencia con la vida social", el grupo de 10 horas tenía una media mucho mas alta que el grupo de 5 horas $(t=17,99$, $\mathrm{p}<0,01$ ). (tabla II). Las mujeres con mas de 30 años no tenían puntuaciones mas altas en el EDQ total $($ media $=99,8)$ que las mujeres menores de 30 años (media $=97,9, t=1,75$, NS). Las mujeres con mas de 40 años contaban con puntuaciones mas altas en el EDQ total $($ media $=101,5)$ que las mujeres por debajo de los 40 (media $=97,9, \mathrm{t}=2,74, \mathrm{p}<$ 0,001). Las mujeres con mas de 50 años no tenían puntuaciones mas altas en el EDQ total $($ media $=100,6)$ que las mujeres menores de 50 años $($ media $=98,3, \mathrm{t}=1,15, \mathrm{NS})$.

\section{Análisis de regresión}

En una regresión múltiple paso a paso con las horas semanales de ejercicio como variable dependiente, se consideraron teóricamente predictores las siguientes variables: edad, BMI, estado civil y los 8 factores EDQ. El modelo final era significativo $(\mathrm{F}(8$, $1125)=104,18, \mathrm{p}<, 001)$. Los dos predictores mas fuertes de alta actividad física fueron: "la interferencia con la vida social" (std.beta $=0,50, \mathrm{t}=17,17, \mathrm{p}<, 001)$ y los "síntomas de abstinencia" (std.beta $=0,20$, t $=6,75, \mathrm{p}<, 001)$. Las variables que tenían validez predictiva fueron bajo "ejercicio por razones sociales" $($ std.beta $=-0,12, \mathrm{t}=$ $4,85, \mathrm{p}<, 001)$, "comportamiento estereotipado" ( std.beta $=0,08, \mathrm{t}=3,38, \mathrm{p}<, 01$ ), "ejercicio por razones sociales" ( std.beta $=$ $0,08, \mathrm{t}=3,27, \mathrm{p}<, 01)$, bajo "insight del problema" ( std.beta $=-0,09, \mathrm{t}=-3,24, \mathrm{p}<$, 01 ), baja "gratificación positiva" (std.beta $=$ $-0,09, \mathrm{t}=-3,15, \mathrm{p}<, 01)$ y bajo "ejercicio por razones de peso" ( std.beta $=-0,08, \mathrm{t}=$ $2,97, \mathrm{p}<, 01)$. La combinación de todas las variables significaron un $43,1 \%$ de varianza. La variables "edad", "BMI" y "estado civil" no pasaron el criterio de entrada.

\section{Discusión}

Los resultados de este estudio muestran una fuerte relación entre las horas semanales de actividad física y las características de la dependencia del ejercicio basadas en el EDQ. Las mujeres con muchas horas semanales de ejercicio físico difieren en relación con el EDQ de las mujeres con menos horas de actividad física semanal. Se han estudiado diferentes formas de actividad física en un mismo grupo. Nos hemos centrado en las altas puntuaciones en el EDQ y en la posible dependencia del ejercicio. Nos podríamos preguntar hasta qué punto algunas mujeres con una alta actividad física en este estudio, están comprometidas de forma negativa en su entrenamiento y actividades físicas. Sin embargo, hemos 


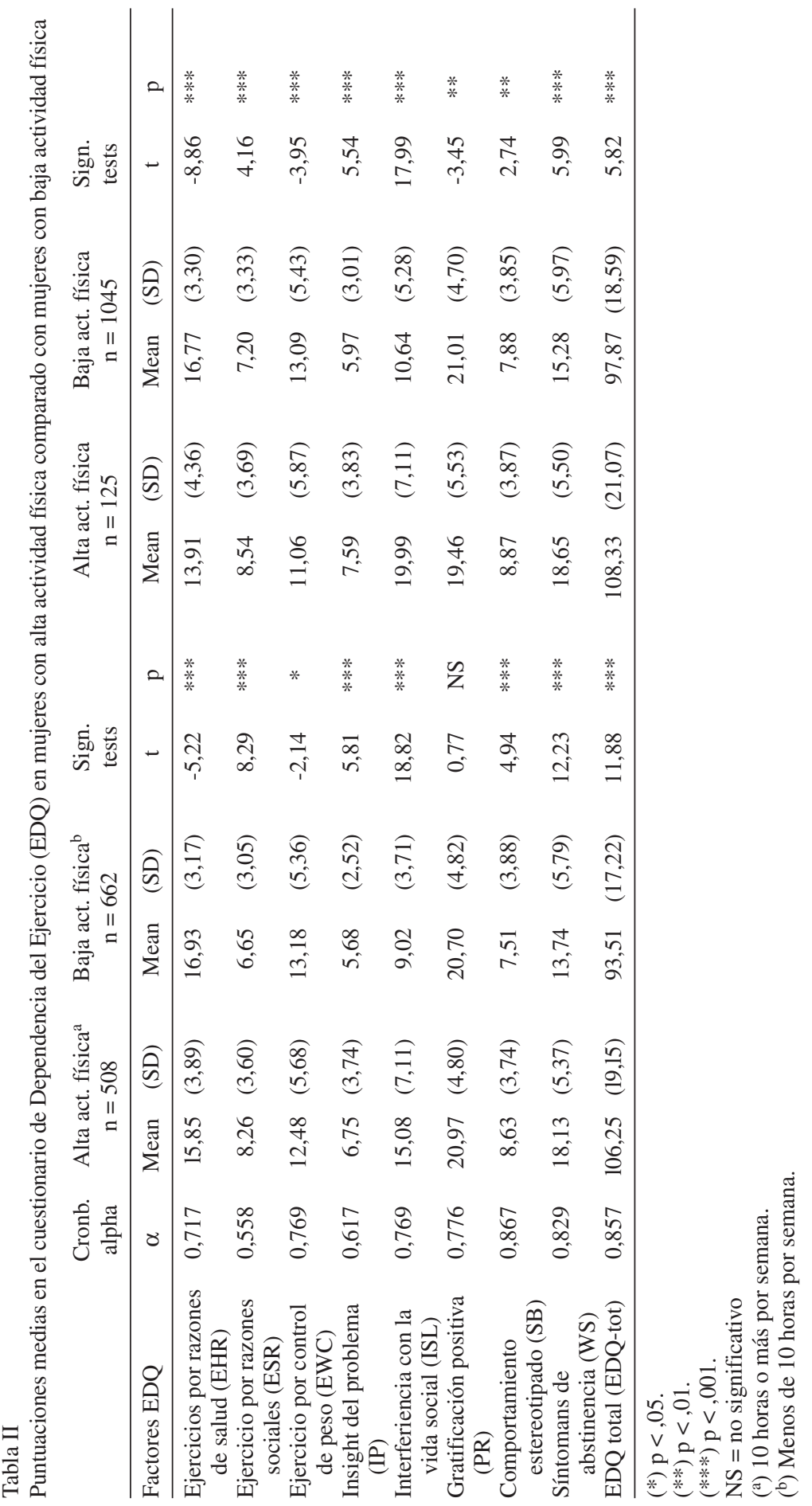


decidido describirlas como posibles mujeres dependientes del ejercicio basado en el EDQ. Podría pensarse de este grupo que se tratase de un grupo de riesgo, cuyos incrementos semanales de ejercicio podrían tener efectos negativos sobre su salud somática y mental.

Ogden et al. (1997) afirmaron como existe una necesidad por encontrar un criterio en el EDQ que permita diferenciar entre dependencia y no dependencia del ejercicio. Hailey y Bailey (1982) han sugerido la "Escala de Adicción Negativa” (NAS), para la determinación de criterios que sirvan para caracterizar esta dependencia negativa. Hauck y Blumenthal (1992) han criticado los métodos utilizados en estudios sobre ExD hasta el año 1992. Ellos han señalado como la mayoría de los estudios se han centrado en el estudio de los diferentes niveles de ExD entre corredores, dejando de lado otros deportes. Según Hauck y Blumenthal, las investigaciones necesitan de métodos mas objetivos con el fin de valorar la conducta de ejercicio, y desde luego, los modelos teóricos deberían desarrollarse y comprobarse de forma empírica. Yates et al. (1994) están de acuerdo con Hauck y Bluementhal, y enfatizan sobre la necesidad de una distinción mas clara entre "adicción" y "compulsión", en otras palabras, entre dependencia y comportamiento compulsivo-obsesivo.

\section{Metodología}

El grupo de estudiantes era representativo de cuatro universidades de Noruega situadas en diferentes áreas geográficas. Podría haber sido mejor que el cuestionario se hubiera enviado a las respectivas casas de cada uno de los estudiantes, con el fin de proporcionar una tasa de respuesta mas alta, pero ello no fue posible debido a los escasos recursos financieros y al poco tiempo disponible. Por otro lado la muestra de este estu- dio constituye una representación limitada de una población normal de mujeres noruegas físicamente activas con edades comprendidas entre 18 y 60 años de edad, ya que la mayoría de las mujeres se encontraban entre los 20 y los 40 años de edad. Además, el 20,6\% ( $\mathrm{N}=252)$ eran trabajadoras y el $33,6 \%(\mathrm{~N}=410)$ habían dado a luz.

Es importante tener en cuenta que estos resultados se basan únicamente en datos autoinformados. Las mujeres con actividad física alta, no necesariamente tienen una relación problemática con el ejercicio físico. Estos individuos podrían sin embargo, conseguir altas puntuaciones en el EDQ. Si no tienen una relación de dependencia con la actividad física y ejercicio, las altas puntuaciones podrían significar alguna otra cosa mas. Ogden et al. (1997) han afirmado que el EDQ podría ser una herramienta útil para categorizar perfiles de atletas y para determinar los factores relacionados con el éxito y el alcance de objetivos.

\section{Interpretación de los resultados}

En este estudio resultó interesante saber que la mujer que hacía ejercicio durante 10 horas o mas a la semana no difería de las otras en cuanto a su estado civil. Este hallazgo parece diferir de los de Dishman (1985) quien aseguraba que algunos individuos dependientes del ejercicio ponían en riesgo su vida social, su matrimonio y sus amistades mas cercanas con el único fin de poder hacer ejercicio. El grupo de las 10 horas parecía ser mas alto y pesado que las mujeres con actividad física baja, y consecuentemente, nosotros encontramos pequeñas diferencias en sus BMI. Esto en parte, podría deberse a una alta desviación estándar en cuanto al peso en el grupo de las 10 horas. Un descubrimiento de interés es que los valores del BMI en los gru- 
pos con actividad física alta, muestran que esas mujeres no se hallan bajas de peso.

Este estudio sugiere que los dos predictores mas importantes en cuanto a la alta actividad física son que el ejercicio afecte a la vida social, y experiencias atléticas distintas del síndrome de abstinencia. Además, los grupos de alta actividad física que lo hacen en su mayoría por razones sociales, tienen una mayor percepción de que el ejercicio físico puede ser un problema potencial, mostrando un comportamiento mas estereotipado. Estos hallazgos parecían ser mas positivos para aquellos que ejercían 10 horas o mas a la semana, indicando la existencia de una relación entre las horas de entrenamiento y la dependencia del ejercicio basado en el EDQ. Una aspecto interesante es que el grupo de las 10 horas mostraba como el ejercicio interfería en su vida social de forma mayor de lo que ocurría con el grupo de las 5 horas. Uno podría pensar que se debía simplemente al número de horas dedicadas al ejercicio físico, sin embargo parece razonable pensar que estos individuos podrían estar preocupados con pensamientos compulsivos y con sentimientos concernientes a la actividad física y al ejercicio. Parece darse un cierto insight o percepción subjetiva del problema así como una cierta experiencia de síntomas de abstinencia cuando se les priva del entrenamiento físico que venían realizando.

En ambos grupos de alta actividad física, es decir en los grupos de 5 y 10 horas se encontraron puntuaciones menores en cuanto a las "razones de salud" y menos en lo referente al "control de peso" que aquellos con baja actividad física. Una observación interesante fue que el grupo de 10 horas contaba con medias mas bajas en estos factores. Esto quiere decir que el tema de la salud era poco prioritario entre las mujeres que realizaban gran actividad física, existiendo otros factores con motivaciones mas fuertes. Para aquellas que realizaban actividad física ocasional, el motivo de una mejor salud podría ser el factor mas significativo. Esto fue confirmado por Anshel (1991) quien sugirió cuatro características en las personas dependientes del ejercicio. Según Anshel, en los dependientes del ejercicio la preocupación y el estrés se da antes de empezar la sesión de entrenamiento y los signos de depresión, ansiedad y agresión, se manifiestan una vez que la sesión de entrenamiento no se realiza. Además experimentan mayores emociones positivas una vez realizada la actividad física, y parecen ignorar los daños físicos y la enfermedad.

Dishman (1985) afirmó que el síndrome de dependencia del ejercicio existe cuando resulta negativo para la salud de uno y cuando los atletas continúan con su ejercicio a pesar de las razones médicas, sociales y de trabajo que les obligarían a parar de hacerlo. Polivy (1991) ha apoyado esta conclusión. En este estudio, la alta actividad física obtenía mayor puntuación en "interferencias con la vida social", pero también en "por razones sociales". La vida social de estas personas podría restringirse al mero deporte, siendo de poca importancia su actividad en otras areas sociales. Quizás las mujeres con alta actividad física se vean a sí mismas como individuos sociales cuando realizan ejercicios físicos, y es por ello por lo que tienen altas puntuaciones en "ejercicio por razones sociales". Sin embargo, se podría también pensar que su nivel de ejercicio interrumpe sus vidas sociales. Un dato interesante era que la mujer de entre 40 y 50 años de edad tenía puntuaciones mas altas en el EDQ total. Estas mujeres se encontraban cercanas a la edad de la menopausia y al cese en la producción de estrógenos, situación que podría llevarles a cambios psicológicos y fisiológicos. El efecto de esto podría llevarles a una excesiva adherencia al ejercicio y a la actividad física. Sin embargo, en 
este estudio, las mujeres que se encuentran en este grupo de edad eran corredoras de grandes distancias que necesitaban realizar una gran cantidad de ejercicios semanales para estar en forma. Esto podría explicar las altas puntuaciones en el EDQ entre las mujeres de 40 a 50 años de edad.

Un individuo con dependencia del ejercicio podría llevarle a asistir a programas de entrenamiento como consecuencia de su aislamiento del medio social. El ejercicio es de importancia por si mismo, mas que por la compañía de los otros con los que se realiza. El aerobic y las pesas suponen la presencia de otros individuos, aunque el contacto entre los participantes puede ser todavía limitado. El ejercicio se realiza para suprimir las emociones negativas y para dar nuevas soluciones a aquellos problemas que puedan aparecer. La expresión "cuando el ejercicio es sentimiento" podría ser de utilidad para ilustrar el balance entre la ocultación de los sentimientos de uno y su expresión.

\section{Conclusiones}

El objetivo de este estudio ha sido el de valorar la puntuación EDQ entre las mujeres noruegas con actividad física. La principal conclusión fue que aquellas mujeres que realizaban una gran cantidad de horas de ejercicio físico semanal se diferenciaban muy claramente en cuanto a las características de dependencia del ejercicio basadas en el EDQ, de aquellas otras mujeres cuya actividad física era menor. El concepto de dependencia del ejercicio físico necesita de una mayor clarificación. Podría considerarse a la adherencia al ejercicio y la regularidad como características necesarias, pero no suficientes de la dependencia del ejercicio, sin embargo el resolver este aspecto disfuncional es todavía algo que se encuentra en proceso de discusión.
Un reto para los equipos deportivos y para los estudios relativos a las tareas de entrenamiento sería el poder ayudar a las personas con una posible dependencia del ejercicio. El mundo del deporte organizado es conocido por tener planificado sus programas de ejercicios específicos así como las horas necesarias de entrenamiento. Un aspecto positivo de esto, es que la mujer físicamente activa podría experimentar un medio social saludable y seguro. Sin embargo, en las mujeres que tienen poco control sobre sus ejercicios, la rutina rigurosa podría dar lugar a comportamientos que conlleven una posible dependencia del ejercicio. Un objetivo razonable para los atletas, entrenadores, terapeutas y profesionales de la salud, sería el de asegurar la adherencia al ejercicio eliminando al mismo tiempo los aspectos negativos propios de una adicción o dependencia.

\section{Reconocimientos}

Los autores gustan expresar su aprecio al Prof. Dana Flanders por su ayuda en cuanto a la metodología y a Cathriona Turner Parker por su lectura de las pruebas de este trabajo.

\section{Bibliografía}

ADAMS, J., KIRKBY, R.J. Exercise dependence: a review of its manifestation, theory and measurement. Sports Med Train Rehab, 8, 265-276, 1998.

American Psychiatric Association. Diagnostic and statistical manual of mental disorders. Fourth Edition (DSMIV). Washington DC: APA, 1994.

ANSHEL, M.H. A psycho-behavioural analysis of addicted versus non-addicted male and female exercisers. J Sport Behav, 2, 145-154, 1991.

BAEKELAND, F. Exercise deprivation: Sleep and psychological reactions. Arch. Gen. Psychiat., 22, 365-369, 1970.

BABR, R., VILBERG, A., OPSTAD, P.K. Overtraining among elite athletes. Tidsskr Nor Laegeforen, 113, 719 722, 1993. 
BENYO, R. The Exercise Fix: How the aerobic athlete's compulsive need for the next workout is self-destructive. Champaign IL: Leisure Press, 1993.

BLAIR, S.N., MCCLOY, C.H. Research lecture: Physical activity, physical fitness and Health. Res $Q$ Exercise Sport, 64, 365-376, 1993

COCKERILL, I.M., RIDDINGTON, M.E. Theory and practice: Exercise dependence and associated disorders: A review. Counsel, Psychol, Quart, 9, 119-129, 1993.

DANIELSEN, $\varnothing$. The new brood arena of sports: Desires and needs of physical activity. Subreport in FAForeport: Sport, leisure and living-conditions, Oslo, 1989.

DAVIS, C. Exercise Abuse. Int J Sport Psychol, 31, 278-289, 2000.

DAVIS, C., FOX, J. Excessive exercise and weight preoccupation in women. Addict Behav, 18, 201-211, 1993.

DAVIS, C., KATZRNAN, D.K., KIRSH, C. Compulsive physical activity in adolescents with anorexia nervosa: A psychobehavioral spiral of pathology. J Nerv Ment Dis, 187, 336-342, 1999.

DISHMAN, R.K Medical psychology in exercise and sport. Med Clin N Am, 69, 123-143, 1985.

DISHMAN, R.K. Exercise Adherence - Its impact on public health. Campaign IL: Human Kinetics Publishers, 1988.

GARNER, D.M., OLMSTED, M.P., BOHR, Y., GARFINKEL, P.E. The Eating Attitudes Test: Psychometric features and clinical correlates. Psychol Med, 12, 871-878, 1982.

HAILEY, B.J., BAILEY, L.A. Negative addiction in runners: A Quantitative approach. J Sports Behav, 5, 150154, 1982.

HAUCK, E.R., BLUMENTHAL, J.A. Obsessive and compulsive traits in athletes. Sports Med, 14, 215-227, 1992.

HOLLANDER, D.B., MEYERS, M.C., LEUNES, A. Psychological factors associated with overtraining: implications for youth sport coaches. J Sport Behav, 18, 3-20, 1995.

HOLLANDER, E. obsessive-compulsive-spectrumdisorders: an overview. Psychiat Ann, 23, 355-358, 1997.

MARLATT, G.A., GORDON, J.R. Relapse Prevention. Guilford: New York, 1985.

McNAIR, D.M., LORR, M., Droppleman, L.F. Profile of mood states manual. San Diego: CA: Educational and industrial testing service, 1971.

OGDEN, J., VEALE, D.M.W., SUMMERS, Z. The development and validation of the Exercise Dependence Questionnaire. Addict Res, 5, 343-356, 1997.

ORFORD, J. Excessive Appetites. A psychosocial view of addictions. Wiley: Chichester, 1987.
PAFFENBERGER, R.S., HYDE, R T. Exercise adherence, coronary heart disease and longevity. Dishman, R.K., (ed.). Exercise Adherence: Its Impact on Public Health. Champaign IL: Human Kinetics Publishers, 1989.

PETRUZELLO, S.J., LANDERS, D.M., HATFIELD, B.D., KUBITZ, K.A., SALAZAR, W. Effects of Exercise on anxiety and mood: A meta-analysis. Sports Med, 11, 143182, 1991.

PIERCE, E.F. Exercise dependence syndrome in runners. Sports Med, 18, 149-155, 1993.

PIERCE, E.F., McGOWAN, R.W, LYNN, T.D. Exercise dependence in relation to competitive orientation in runners. J Sport Med Phys Fit, 33, 189-193, 1993.

POLIVY, J. Physical activity, fitness and compulsive behaviors. Bouchard, C., Shephard, R.J, Stephens, T., (eds.). Physical Activity, Fitness and Health: International Proceedings and Consensus Statement. Campaign IL, Human Kinetics Publishers: 888-897, 1991.

SKÅRDERUD, F. Nervøse spiseforstyrrelser (Nervous eating disorders). Universitetsforlaget: Oslo, 1994.

SZABO A. The impact of exercise deprivation on wellbeing of habitual exercisers. Aust J Sci Med Sport, 27, 6875,1995

THORNTON, E.W., SCOTT, S.E. Motivation in the committed runner: Correlations between self- report scales and behaviour. Health Promot Int, 10, 177-184, 1995.

VAN ITALLIE, T.B. Health implications of overweight and obesity in the United States. Ann Intern Med, 103, 983-988, 1985.

VEALE, D.M.W. Exercise dependence. Brit JAddict, 82, 735-740, 1987.

VEALE, D.M.W. Psychological aspects of staleness and dependence on exercise. Int J Sports Med, 12, 19-22, 1991.

YATES, A., LEEHEY, K., SHISSLAK, C.M. Running an analog of anorexia? New Engl J Med, 308, 251-255, 1983.

YATES, A., SHISSLAK, C.M., Crago, M., Allender, J. Overcommitment to sport: Is there a relationship to the eating disorders? Clin J Sport Med, 4, 39-46, 1994.

Dirección para correspondencia:

Einar Kjelsås

E-mail: einar.kjelsas@medisin.ntnu.no

Departamento de Psiquiatría y Medicina de la Conducta

Hospital Östmarka (NTNU)

P.O. Box 3008 Lade

NO-7441 Trondheim

NORUEGA 